\title{
PAPR Reduction in OFDM System Using PTS+DCT+SLM Technique
}

\author{
Sweety Narula ${ }^{1}$, Ritesh Beohar ${ }^{2}$ \\ ${ }^{1,2}$ GGITS, Jabalpur, M.P., India
}

\begin{abstract}
In communication system the operation of the transmitter power is limited to some range and if the input signal with an amplitude more than the transmitter power amplifier linear range results in signal distortion. Since now the demand for multimedia data services has grown up rapidly one of the most promising method is considered as the OFDM (Orthogonal frequency division multiplexing).Since the subcarriers, high data rate, ubiquitous coverage with high mobility and many such features not only enhances the usability of the system but has given a major disadvantage too. Peak-to-average-power ratio (PAPR) has highly affected the OFDM system. The high the PAPR in the signal it will occasionally drive high power amplifiers (HPAs) to operate in the nonlinear region of their characteristic curve. Here the nonlinearity can cause loss of orthogonality amongst the carrier causing inter carrier interference (ICI) in signal. In-band and Out-band radiations are also outcomes of the PAPR. In this paper we will implement techniques to low the PAPR.
\end{abstract}

Keywords: PAPR, OFDM, HPA, DCT, SLM, PTS

\section{Introduction}

In this smart world the demand for the multimedia data service has grown drastically with the introduction of the $4^{\text {th }}$ generation wireless communication. Here the number of users are much higher than the spectrum present for the communication. For this we have used OFDM as it gives Bandwidth efficiency, High data rates and is immune to fading marks. It is majorly used in Digital audio Broadcasting (DAB), Digital Video Broadcasting (DVB), Long Tern Evolution (LTE) and much more. The major disadvantages cased are tight frequency synchronization, time offset, peak to average power ratio (PAPR) and channel estimation. OFDM is the base of all 4G wireless communication system since it has a huge capacity of number of subcarriers, high data rate as high as $100 \mathrm{Mbps}$ and ubiquitous coverage with high mobility.

In the OFDM modulation High PAPR is one of the major issues, when $\mathrm{N}$ number of sinusoidal signals get added the peak magnitude will have a value of $\mathrm{N}$ where at some point of time the average might be low due to the interference in the signal or it could also be high due to constructive interference between the signal

Therefore High PAPR signals is undesirable as due to high PAPR it would be an important requirement that a large range of dynamic linearity from the analog circuits which ultimately results in expensive devices and high power consumption making the device less efficient (For e. g. Power amplifier will have to operate with large back-off to maintain the linearity).

In OFDM systems the input sequence needed are to be transmitted and would result in higher PAPR than other techniques. So an input sequence which requires all such carriers to transmit their maximum amplitudes would certainly result in a high output PAPR. Thus when we restrict the possible inputs sequence to smallest set of values then there might be probability that to obtain output signals with low PAPR.

\section{Literature Review}

PAPR is the main issue in the OFDM systems, it degrades the performance of the system when a non-linear HPA is used. It is necessary to use an appropriate PAPR reduction technique in the transmitter side. We have done the detailed analysis of PAPR, there impact definition parameters to analysis PAPR and different methods of PAPR reduction are included. Simulated results are discussed and analyzed.

Many PAPR reduction techniques are proposed in this literature review to reduce the PAPR of the OFDM signal. The division is done in two categories

a) Distortion Based Techniques

b) Non-Distortion Techniques

The techniques which introduces spectral re-growth belong to distortion based are the most straight forward PAPR reduction methods. One of the distortion based techniques is the clipping. It reduces the peak of the OFDM signal by clipping the signal to the desired level, but inspite of its simplicity it introduces both the in-band distortion and the out-of-band radiation. Jean Armstrong proposed an iterative clipping and filtering schemes to constraint the out-of-band radiation.

Another popular distortion based technique is the Companding which reduces the PAPR in the OFDM systems. Wang et al. proposed a scheme based on $\mu$-law companding to reduce the PAPR of the transmitted signal. In $\mu$-law companding the peak value is kept same before and after the companding. It keeps the peak power of the OFDM signal same but the average power after companding increases a lot. This increase in the average power reduces the PAPR in the signal. But a major disadvantage is that the error performance of $\mu$-law companding scheme degrades.

Another scientist named Jiang et al. proposed exponential (EC) function to transform Rayleigh magnitude of the signal into uniform distribution using an exponential function known as "Exponential companding". Exponential 


\section{International Journal of Science and Research (IJSR) \\ ISSN (Online): 2319-7064}

Index Copernicus Value (2013): 6.14 | Impact Factor (2014): 5.611

companding scheme can effectively reduce the PAPR of the OFDM signal but its BER performance also degrades with PAPR reduction. Huang et al. proposed four companding transformation functions to reduce the PAPR of the OFDM signal, which includes: linear symmetrical transform (LST), linear non symmetrical transform (LNST), non-linear symmetrical transform (NLST) and non-linear nonsymmetrical transform (NLNST). It is observed that the performance of the LNST is comparatively better from all the four functions. Aburakhia et al. proposed, Linear Companding Transform (LCT) to reduce the PAPR. Here large and small signals are treated on different scales but they have two inflexion points to achieve more flexibility in designing the companding function, this abrupt change in the edited signal at the infection points degrades the power spectral density hence minimizing the PAPR.

Hou et al. proposed, Trapezoidal Companding which is also an efficient method to reduce the PAPR of the OFDM signal with low BER. Here the Rayleigh distributed magnitude is distributed in a trapezoidal fashion. In TC piecewise function in the three defined intervals of OFDM magnitudes is greatly utilized. Similar to it Jeng et al. proposed another method using the Trapezium distribution based companding (TDBC) here the Rayleigh distribution is based on linear trapezium. All the above techniques are types of the distortion based techniques. The main disadvantage here is the shape of the signal is compromised for PAPR reduction which effects the performance of the signal as a whole.

The second type of Techniques are the Non-Distortion based PAPR reduction techniques. Here the shape of the signal will not be compromised and no spectral re-growth takes place. One of the most basic and simplest technique of the non-distorted is the Coding Technique (CT). It can efficiently reduce the PAPR of the signal but the data loss can also happen in this technique.

Other two techniques of distortionless PAPR reduction are partial transmit sequence (PTS) and the selective mapping (SLM).In the PTS technique the subcarriers are portioned into multiple disjoint sub parts and the rotating phase factor is multiplied with all the sub parts and are combined to achieve a signal with lowest PAPR whereas in SLM the parallel data signal of $\mathrm{N}$ length is multiplied by a set of $\mathrm{U}$ phase vectors of $\mathrm{N}$ length the set is predetermined and it generates $U$ alternative signals. Out of them the least PAPR signal is selected for transmission. In both of the schemes the information about the phase factors by which these sub blocks/data symbols are multiplied, needs to be conveyed to the receiver and it is known as side information (SI). The SI has the highest importance because it is used to recover the original data signal. If SI gets corrupted then entire OFDM symbol block can be damaged and error performance of SLMand PTS-OFDM system degrades severely.

In PTS if the number of sub blocks increases then there is an increase in the computational complexity also but it also increases the amount of SI which is conveyed to the receiver at the receiving end.If the SI is increased the chances of data loss in the signal also increases. Similar is the case of SLMOFDM, here if the number of alternative signals are increased then the number of bits for encode also increases and hence results in data loss. Since the SI bits are extremely important for the data recovery and it is important to give some redundant bits to ensure accurate SI recovery rate. But aging if will affect the data and loss may occur.

There are multiple schemes which are proposed for embedding the SI in the OFDM systems.It embed SI in the OFDM signal without using an extra bit. When the signal reaches the receiver $\mathrm{Si}$ is extracted from the received OFDM signal. It is then decoded to obtain the information about the phase factor used to reduce the PAPR. The converted signal is then multiplied by the reciprocal of recovered phase factors, due to which the computational complexity at the receiving end gets increased. In multiple techniques a major disadvantage is that the SI detection at lower values of SNR is very poor and it leads to error performance of the OFDM system degrades severely.

Another system MPSM-PTS is proposed by Zhou et al. it extends the QPSK constellation points to disjoint points of 16-QAM constellation and eliminates the requirement of side information. The scheme which is completely free from the SI is MPSM-PTS scheme. When the signal is received the extraction of the SI is not needed and the receiver structure of the scheme proposed in is computationally less complex.

In systems like LTE OFDM is used as downlink and the mobile station act as receivers. Here the mobile station have only a handful of resources therefore a PAPR reduction scheme with less computational complexity at receiving end will be more beneficial.As reviewed the schemes proposed are computationally complex receiver in comparison to the schema proposed. Hence MPSM-PTS scheme is a viable choice for PTS-OFDM system.

OFDM is sensitive to small carrier frequency offset in between transmitter and receiver carrier frequencies can disturb the orthogonality of the subcarriers and causes ICI. The ICI interference degrades the overall performance of the system. It is generally characterized by carrier to interference ratio (CIR).

Various ICI cancellation techniques have been proposed in the literature to eliminate the effect of ICI, these include ICI self-cancellation, New ICI self-cancellation, General ICI self-cancellation scheme, ICI conjugate cancellation scheme, General phase rotated conjugate transmission ICI cancellation scheme etc.

\section{Proposed System}

Different techniques are developed to reduce the PAPR value from the transmitter medium to improve the efficiency of the system and remove the noise from the transmission line. Now we will see those techniques which we propose for better output.

\section{Partial Transmit Sequence}

Partial Transmit Sequence (PTS) is one of the most efficient techniques to diminish PAPR. In this scheme original OFDM signal is divided into number of sub-blocks. Then

\section{Volume 4 Issue 11, November 2015}




\section{International Journal of Science and Research (IJSR) \\ ISSN (Online): 2319-7064}

Index Copernicus Value (2013): 6.14 | Impact Factor (2014): 5.611

phase rotation is added to develop number of candidate signal and choose one with lowest PAPR.

In the PTS Technique, the input symbol sequence is partitioned into a number of disjoint symbol subsequences. IFFT is then applied to each symbol subsequence and the resulting signal subsequences are summed after being multiplied by a set of distinct rotating vectors.

Let input data blocks $\mathrm{X}=\left\{X_{K}\right\}$, where $(k=1,2 \ldots N-1), \mathrm{N}$ is number of sub-carriers. Make $M$ is the frequency domain (FD) data sequences, $X^{\varepsilon}(\varepsilon=1,2, \ldots M)$, by multiplying phase sequences

$X^{\varepsilon}=\left\{P_{K}^{\varepsilon}\right\}(K=0,1,2, \ldots N-1)$

With $\mathrm{X}$ elements provide following results

$X^{\varepsilon}=\left[P_{0}^{\varepsilon} X_{0}, P_{1}^{\varepsilon} X_{1} \ldots \ldots P_{N-1}^{\varepsilon} X_{N-1}\right]$

$\varepsilon=(1,2, \ldots, M)$

Where $P_{K}^{\varepsilon}=\exp \left(j \varphi_{K}^{\varepsilon}\right), \varphi_{K}^{\varepsilon}$ is uniformly distributed in $[0$, $2 \pi]$.

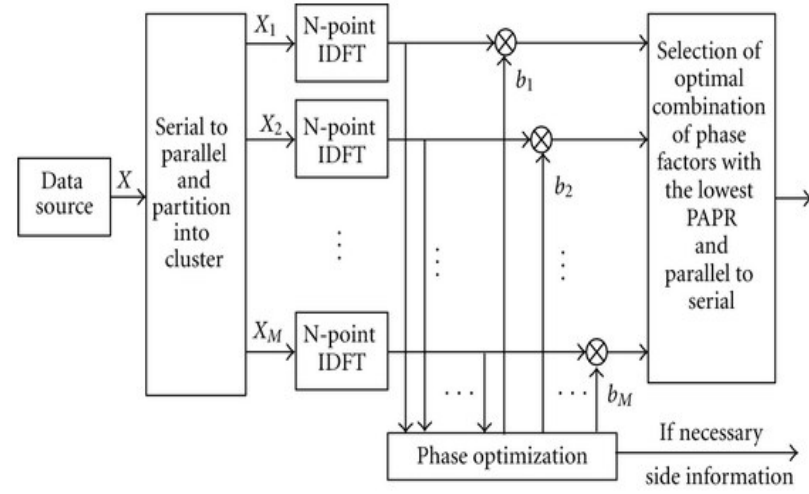

Figure 1: Block diagram of conventional PTS scheme.

To get $\mathrm{M}$ candidates' time domains using IDFT

$X^{\varepsilon}=I D F T\left\{X^{\varepsilon}\right\}_{s} \varepsilon=(1,2, \ldots, M)$

All the candidates have same information $\mathrm{x}$ provide different PAPRs. One with smallest PAPR in $X^{\varepsilon}$ is selected for transmission.

\section{Selective Mapping}

Selective Mapping is promising technique to mitigate PAPR in OFDM system. Fundamental idea behind scheme is phase rotation. Signal with low PAPR is selected from different independent phase sequences that have same information at transmitter.

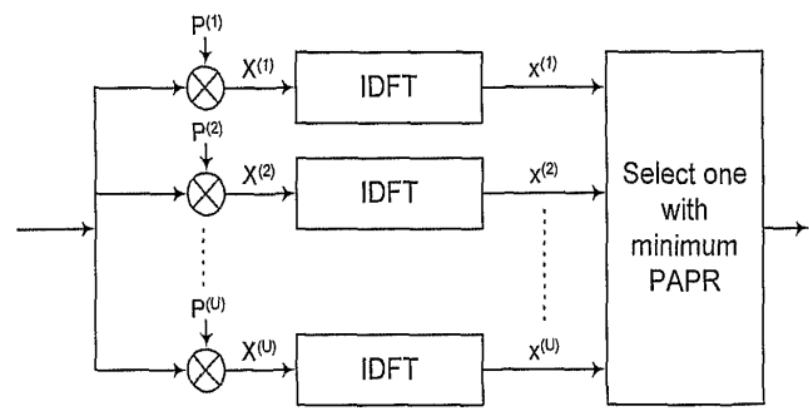

Figure 2: block diagram of SLM technique

\section{$X=\left[X_{0}, X_{1}, X_{2}, \ldots, X_{N-1}\right]^{T}$}

When multiply with independent phase sequence results $P^{u}=\left[P_{0}^{u}, P_{1}^{u} \ldots X_{N-1}^{U}\right]^{T}, u=(1,2, \ldots, U-1)$

$\mathrm{U}=$ number of phase sequence

Keep length of input data and phase sequence same. Then get time domain signal by applying IFFT we get data block with different PAPR value and phase sequence.

$X^{W}=\left[X_{0}^{u}+X_{1}^{u}+\ldots \ldots X_{N-1}^{U}\right]^{T}$

Select one with low PAPR and transmit. CCDF is used to measure the probability that the PAPR of a certain data block exceeds the given threshold. CCDF of PAPR in SLM will be

$P(P A P R>P A P R O)=\left(1-\left(1-e^{-P A P R O}\right)^{\alpha N}\right)^{\mathbb{U}}$

$\mathrm{N}=$ no. of sub-carrier

$N_{\text {UFT }}=\mathrm{N}$-point IFFT operation

$\mathrm{U}=$ independent phase sequence

PAPR0 $=$ threshold value

$\alpha=$ oversampling factor

\section{DCT-SLM:}

The main idea of the scheme is to use a combination of two appropriate methods. One is the DCT matrix transform technique and the other is the SLM technique. The transmitter block is showed in Figure 1. In the transmit end, the data stream is firstly transformed by DCT matrix, then the transformed data is processed by the SLM unit. If data block passed by DCT matrix before IFFT, the autocorrelation coefficients of IFFT input is reduced, then the PAPR of OFDM signal could be reduced.

In this DCT matrix use after SLM to further reduce the PAPR of signal. In his fashion, the autocorrelation of the signal, which has been processed by SLM, is reduced by DCT matrix transform. The PAPR of fine output signal is further reduced. The block of transmitter is showed in Figure 2.

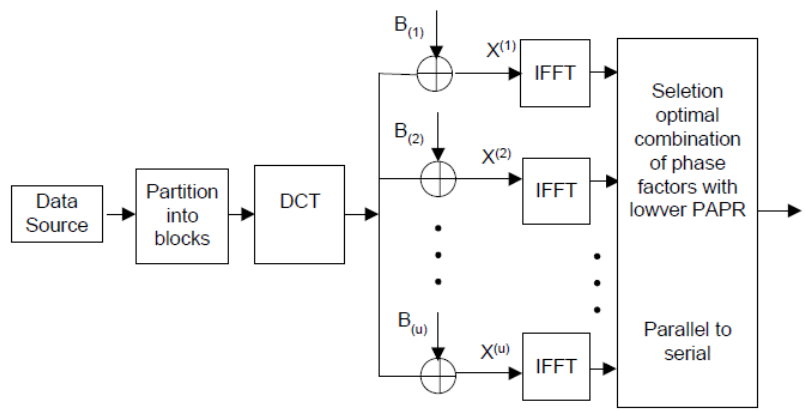

(a)

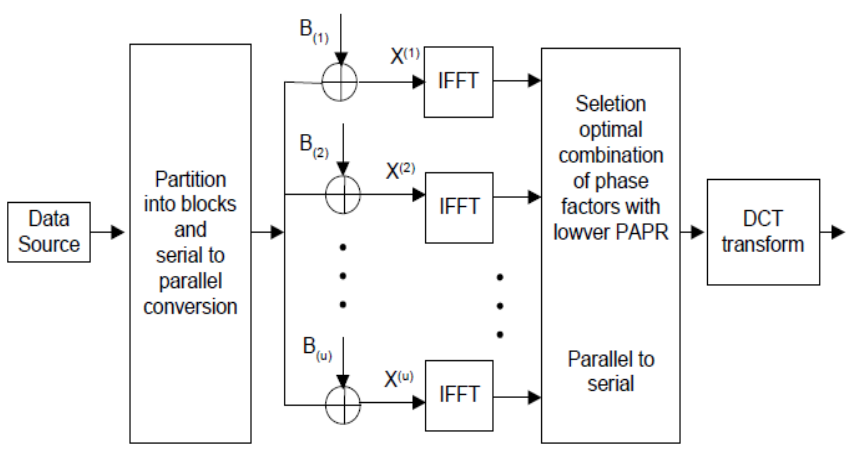

(b)

Figure 3: Block Diagram of DCT-SLM scheme

Let input data blocks be 


\section{International Journal of Science and Research (IJSR) \\ ISSN (Online): 2319-7064}

Index Copernicus Value (2013): 6.14 | Impact Factor (2014): 5.611

\section{Implementation}

To implement such active set approach for PAPR reduction signal we generate symbols of length 512 and 1024 samples which contain 301 or 601 subcarriers respectively. The implementation techniques have been performed using the MATLAB. The parameters used for the analysis are summarized in table 1:

\begin{tabular}{|l|l|}
\hline Parameters & Values \\
\hline System Sub-carrier & 512,1024 \\
\hline MIMO Scheme (Tx $\times \mathrm{Rx})$ & $(2 \times 2)$ \\
\hline Oversampling factor (L) & 6 \\
\hline Modulation scheme & QPSK \\
\hline Phase factor & $1,-1, \mathrm{j},-\mathrm{j}$ \\
\hline Clipping Ratio (CR) & 4 \\
\hline Route numbers used in SLM method & $\mathrm{M}=8$ \\
\hline $\begin{array}{l}\text { Number of sub-blocks used in PTS } \\
\text { methods }\end{array}$ & $\mathrm{V}=8$ \\
\hline Random OFDM symbols generated & 1000 \\
\hline \multicolumn{2}{|l|}{ Table 1. Parameters used in clipping and filtering, PTS, SLM and algorithm }
\end{tabular}

It summarizes the parameters used for PAPR reduction. Here the number of sub carriers is 512 and 1024. A system MIMO-OFDM modulated with QPSK is used and the simulated results are calculated. The complementary cumulative distribution function (CCDF) of the PAPR for the transmitted signal are plotted after each methods reducing PAPA used. The flow used for implementation is as follows:

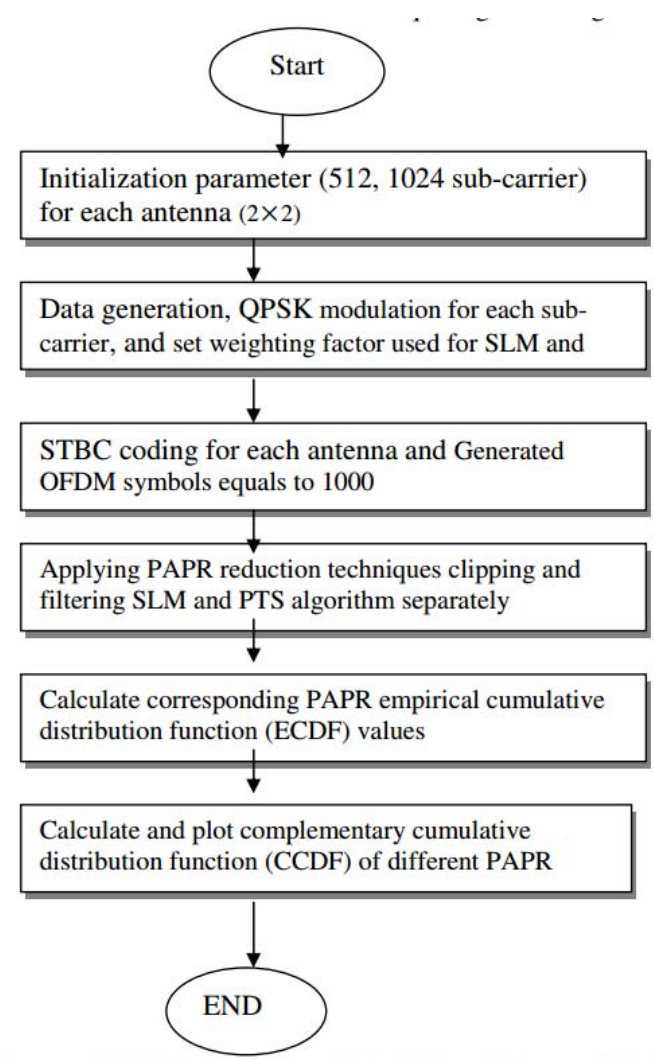

Figure 4 PAPR Reduction algorithm techniques clipping and filtering SLM and PTS

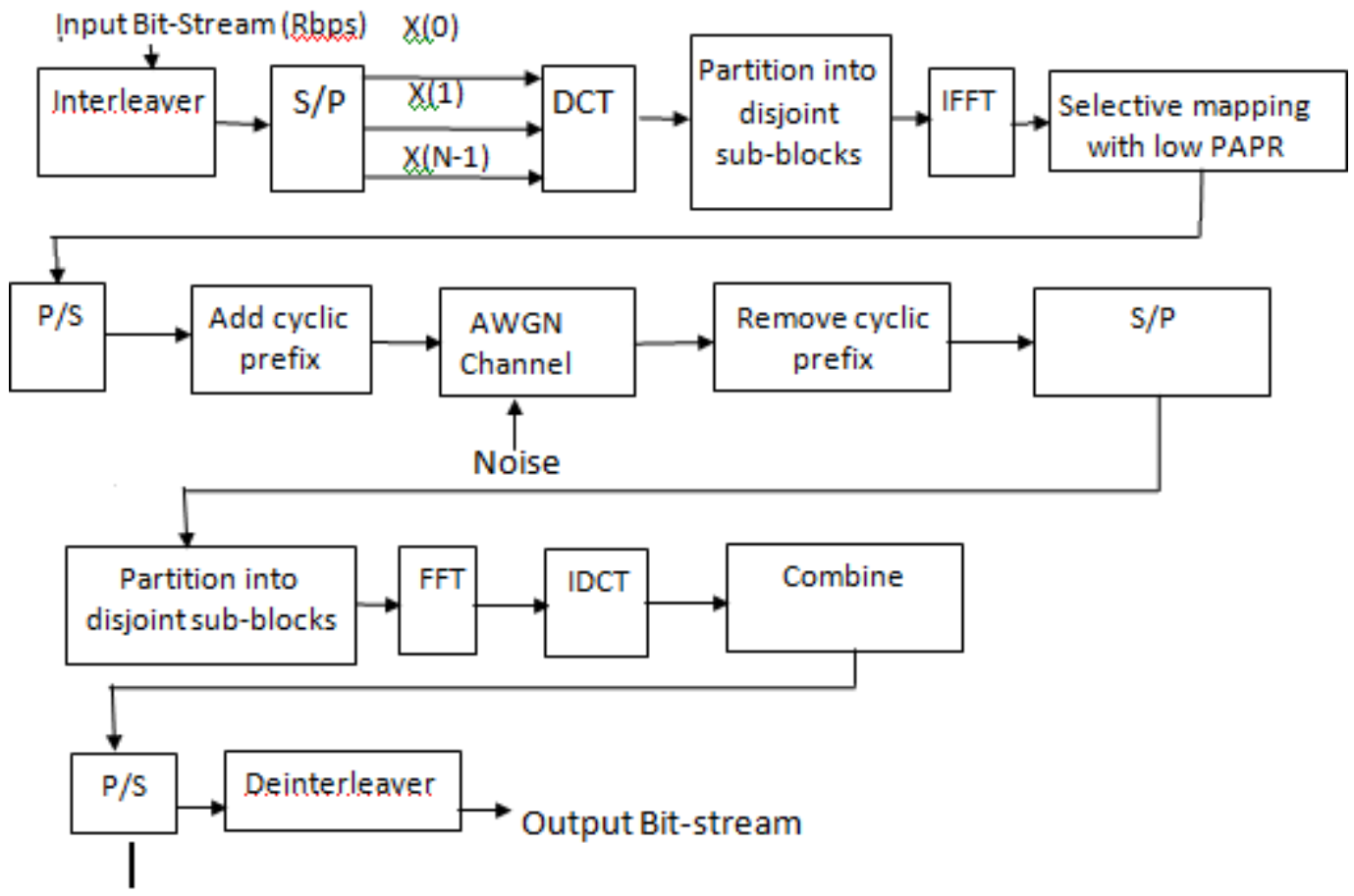

Figure: Block Diagram of the Proposed System

\section{Result}

\begin{tabular}{|c|l|l|l|}
\hline No. of Frames & SNR=6 & SNR=15 & SNR=30 \\
\hline \multirow{2}{*}{5} & 14.40951 & 14.41069 & 14.41862 \\
\hline \multirow{2}{*}{} & 14.40571 & 14.40749 & 14.40709 \\
\hline & 14.40726 & 14.40707 & 14.40871 \\
\hline & 14.40866 & 14.40852 & 14.41093 \\
\hline 14.40879 & 14.41009 & 14.40647 \\
\hline
\end{tabular}

Figure: PAPR in OFDM without using DCT
This figure shows the PAPR rate of the OFDM system when none of the reduction techniques is applied. When the SNR is 6 the PAPR would be 4.41 approx. The same rate of the PAPR can be seen when the SNR is 15 and 30 respectively. This PAPR is comparatively very high and can damage the message. 
International Journal of Science and Research (IJSR)

ISSN (Online): 2319-7064

Index Copernicus Value (2013): 6.14 | Impact Factor (2014): 5.611

\begin{tabular}{|c|l|l|l|}
\hline No. of Frames & SNR=6 & SNR=15 & SNR=30 \\
\hline \multirow{3}{*}{5} & 10.80066 & 10.82571 & 10.41585 \\
\cline { 2 - 4 } & 10.53675 & 10.80333 & 10.17117 \\
\hline \multirow{2}{*}{} & 10.69467 & 10.73077 & 10.78922 \\
\cline { 2 - 4 } & 10.67622 & 10.78052 & 10.51871 \\
\hline & 10.44378 & 10.24012 & 10.76414 \\
\hline
\end{tabular}

Figure: PAPR in OFDM using DCT

The above figure shows the result when DCT is applied. When the DCT is applied when SNR is 6 the PAPR rate is 10.5 approx. After applying this single reduction technique the PAPR is reduced from almost $30 \%$. Which is a good percentage and can reduce the chances of the error in data.

\section{\begin{tabular}{|l|l|l|l|} 
No. of Frames & SNR=6 & SNR=15 & SNR=30
\end{tabular} \begin{tabular}{|l|l|l|}
5.09132 & 5.03693 & 5.30095
\end{tabular} \begin{tabular}{|l|l|l|l|l|}
\hline 5 & 5.19191 & 5.17427 & 5.08330 \\
\hline
\end{tabular} \begin{tabular}{|l|l|l|}
5.10358 & 5.12366 & 5.23656
\end{tabular} \begin{tabular}{|l|l|l|l|}
\hline 5.23051 & 5.35048 & 5.16038 \\
\hline
\end{tabular} \begin{tabular}{|l|l|l|l|}
5.11402 & 5.03517 & 5.17117
\end{tabular}}

Figure: PAPR in OFDM using DCT, SLM and PTS

The above figure shows the result of the system we implemented. Here we would be using three techniques to reduce the PAPR which are DCT, SLM, and PTS. When the SNR value is 6 then the PAPR is nearly 5 . Which is very less compared to the other methods. The PAPR is reduced nearly by $67 \%$, which is a great percentage. Here there would be very less chances of distortion and data loss.

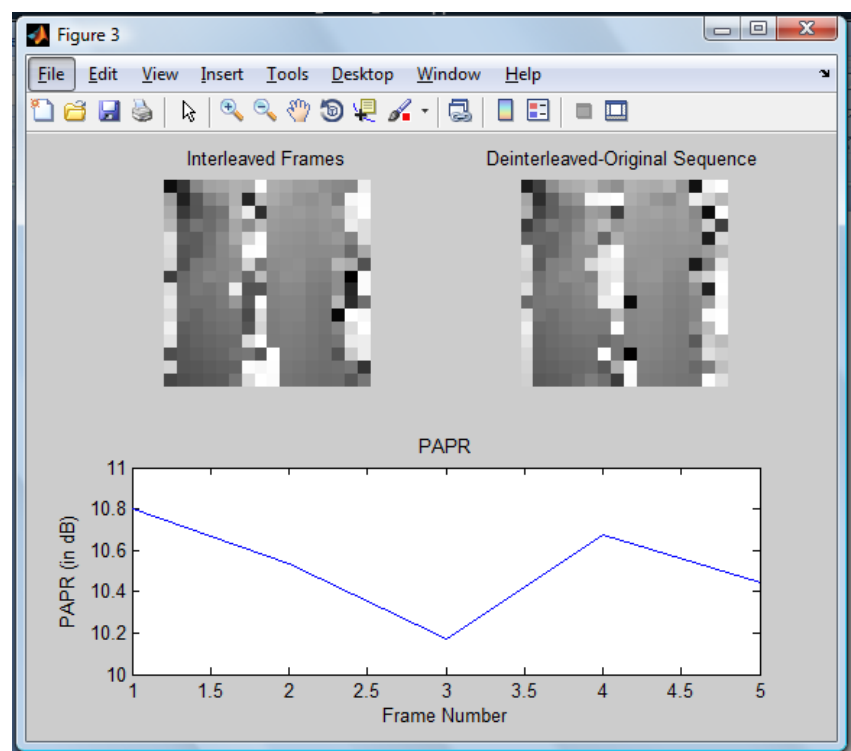

Figure: PAPR in OFDM using DCT

The figure shows the output with the sender frame and the received frame. The PAPR level of the frames are shown below.

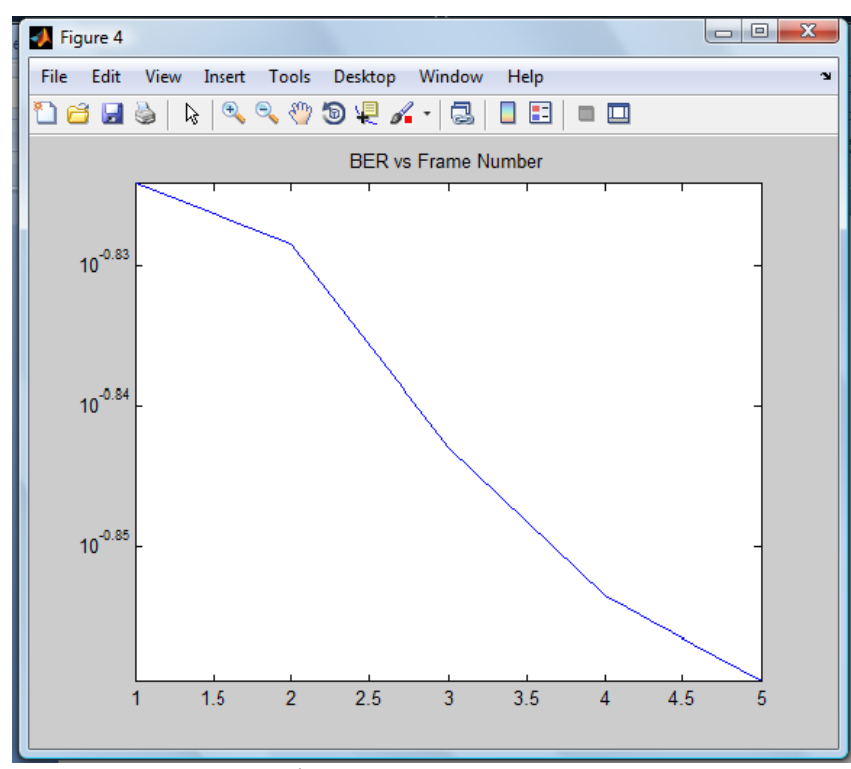

Figure: BER V/s FRAME NUMBER USING DCT

The above figure shows the BER reduction as the frames increases and the PAPR reduction technique is applied to the system. When we apply PAPR reduction technique the BER also degrades.

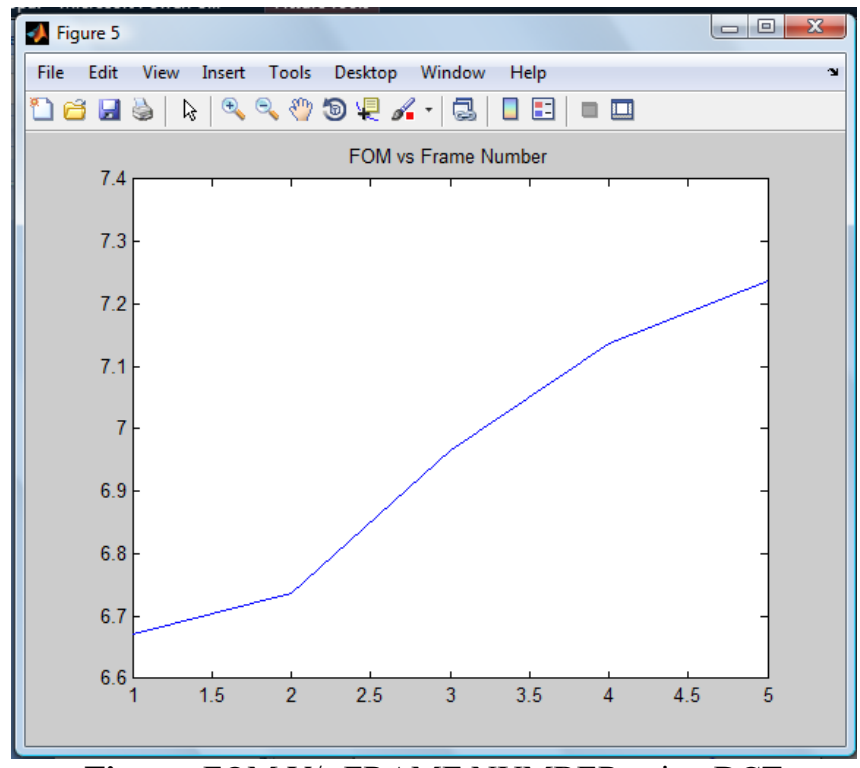

Figure: FOM V/s FRAME NUMBER using DCT

When we apply the DCT i.e. PAPR reduction techniques then the FOM of the signal increases. The graph below without DCT shows that the FOM is comparatively less after applying the reduction technique to the signal. 


\section{International Journal of Science and Research (IJSR) \\ ISSN (Online): 2319-7064}

Index Copernicus Value (2013): 6.14 | Impact Factor (2014): 5.611

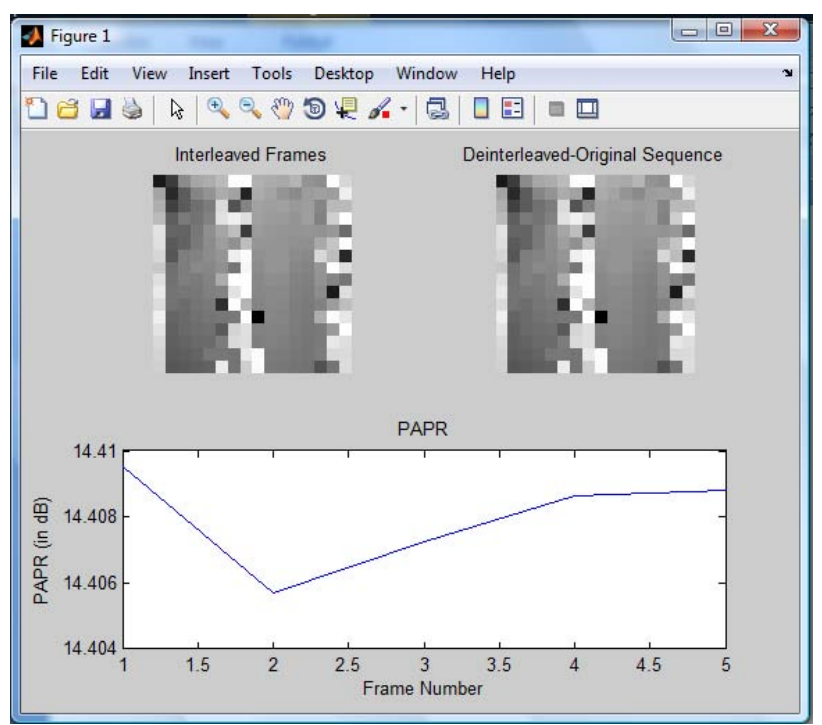

Figure: PAPR in OFDM without using DCT

The above figure shows the shows the sent and received signal frames, the graph below shows that the PAPR in the signal is much higher. And it could lead to data loss.

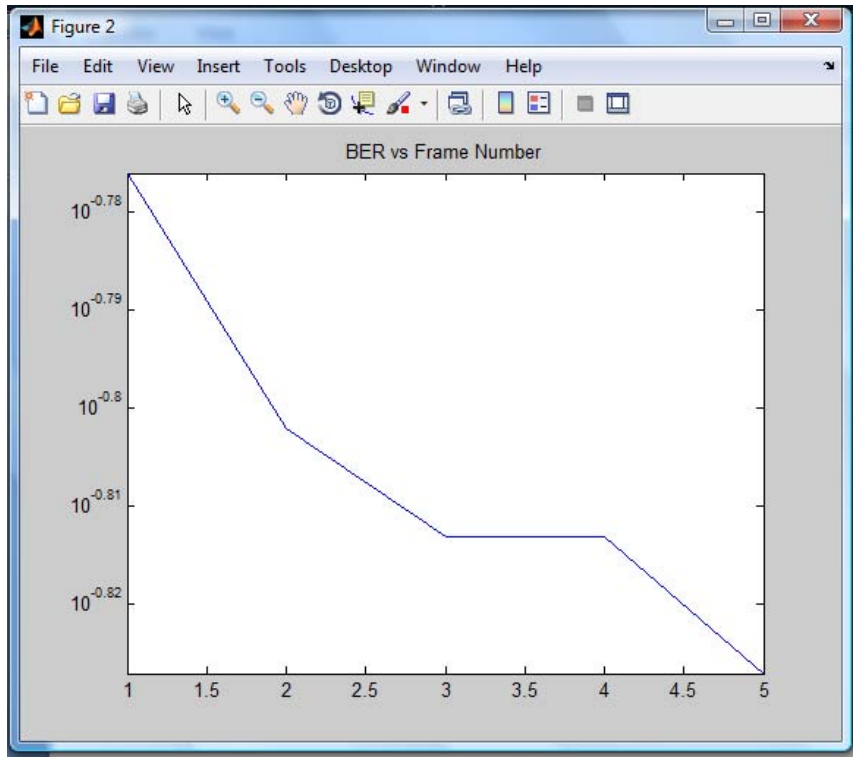

Figure: BER V/s FRAME NUMBER without using DCT

Since the PAPR reduction techniques changes the value of BER. Here compared to the graph using DCT and the proposed system the BER has somewhat greater value then both the systems.

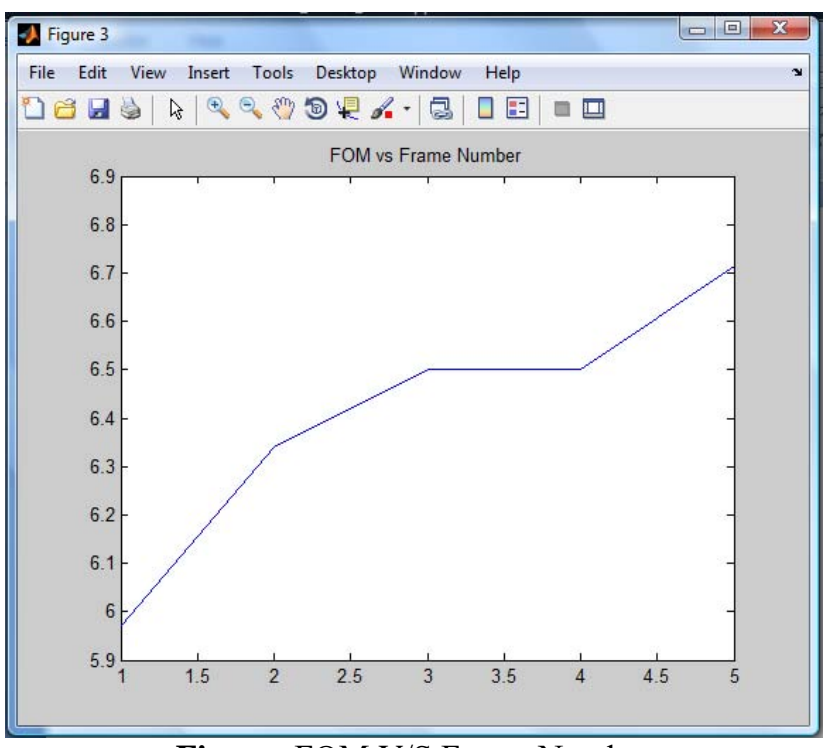

Figure: FOM V/S Frame Number

When no PAPR reduction technique is applied then the FOM of the frames is very low. But when it is applied the end FOM value is then nearly the start FOM value.

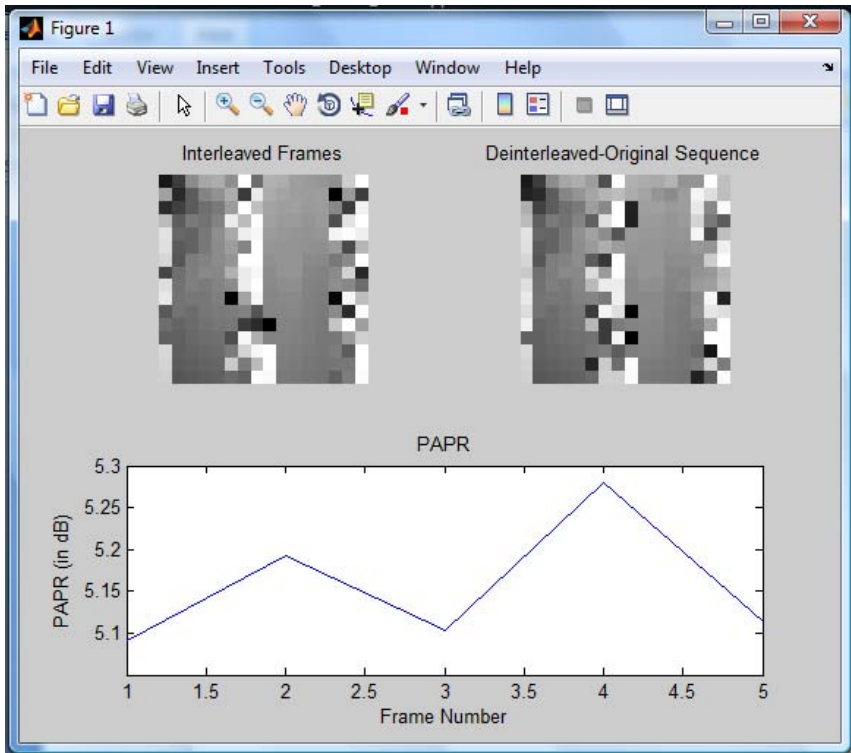

Figure: PAPR in OFDM using DCT, PTS and SLM

The figure shows the output with the sender frame and the received frame. The PAPR level of the frames are shown below. The PAPR is the lowest amongst all the three and hence the system is error free. 


\section{International Journal of Science and Research (IJSR) \\ ISSN (Online): 2319-7064}

Index Copernicus Value (2013): 6.14 | Impact Factor (2014): 5.611

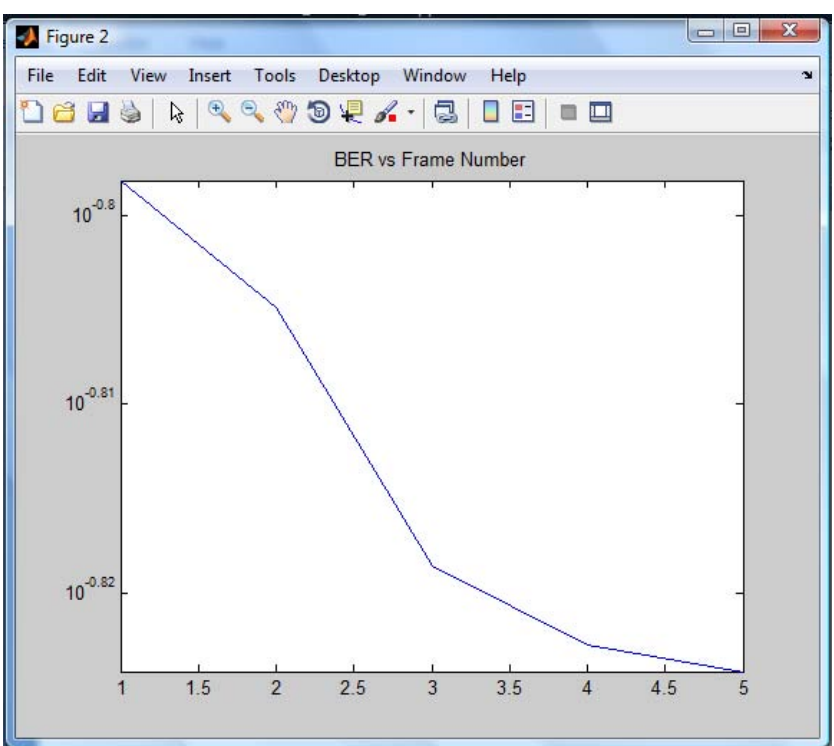

Figure: BER V/s FRAME NUMBER using DCT, PTS and SLM

The above figure shows the BER reduction as the frames increases and the PAPR reduction technique is applied to the system. When we apply PAPR reduction technique the BER also degrades. The degradation is severe here the BER hits nearly nil at 4.5 frame point.

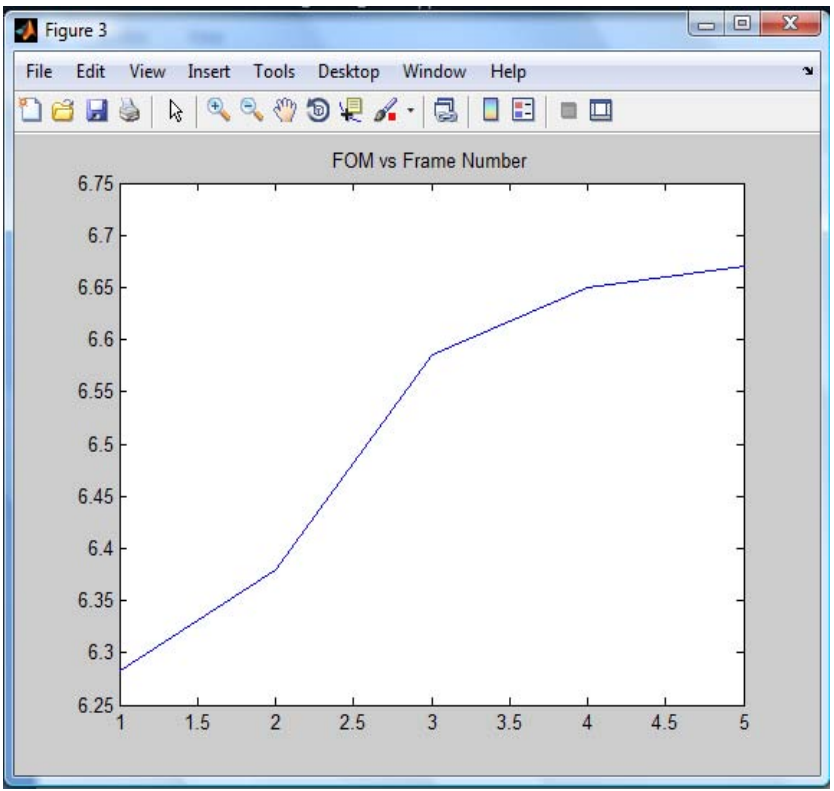

Figure: FOM V/S Frame Number

When no PAPR reduction technique is applied then the FOM of the frames is very low. But when it is applied the end FOM value is then nearly the start FOM value. Here the FOM is in between the lowest and highest start therefore we can say that in the proposed system the FOM is not majorly affected.

\section{Conclusion}

OFDM is considered as one of the most attractive technique for high speed data transmissions and it has a major drawback that is high PAPR, to overcome it we have used three techniques PTS, SLM and DCT-SLM. All these techniques reduces a lot of PAPR from the signal sent without distorting the signal and without major data loss. However we cannot specify that any of the technique is the best to reduce the PAPR from the OFDM system. BER increase and computation complexity are also major factors which decides the appropriate PAPR technique.

\section{References}

[1] "PERFORMANCE IMPROVEMENT IN OFDM SYSTEM BY PAPR REDUCTION" by Suverna Sengar, Partha Pratim Bhattacharya

[2] "PAPR Reduction in OFDM systems" by Prasanta Kumar Pradhan.

[3] "Different Techniques to Reduce the PAPR in OFDM System" by Malhar Chauhan, Saurabh patel, Hardik patel.

[4] "Improved PAPR Reduction in OFDM Systems" by Ashish Goel.

[5] "Comparative Study of PAPR Reduction Techniques in OFDM" by Md. Ibrahim Abdullah, Md. Zulfiker Mahmud, Md. Shamim Hossain, Md. Nurul Islam.

[6] "An Investigation of Peak-to-Average Power Reduction in MIMO-OFDM Systems",Wang Yi Gu linfeng Blekinge Institute of Technology October 2009.

[7] Jiang T., Wu Y., "An Overview: Peak-to-average power ratio reduction techniques for OFDM signals", IEEE Trans. Broadcasting, vol. 54, No. 2, pp. 257-268, Jun. 2008.

[8] Zhou, Y. , Jiang, T., "A Novel Multi-Point Square Mapping Combined with PTS to Reduce PAPR of OFDM Signals without Side Information", IEEE Transaction on Broadcasting, Vol. 55, No. 4, pp. 831835, Dec. 2009.

[9] Armstrong, J., "Peak-to-average power reduction for OFDM by repeated clipping and frequency domain filtering," Electronics Letters, vol. 38, No. 5, pp. 246247, Feb. 2002.

[10] Wang X. B., Tjhung T. T., Ng C. S., "Reduction of peak-to-average power ratio of OFDM system using a companding technique", IEEE Transaction on Broadcasting, vol. 45, No. 3, pp. 303-307, Sept. 1999.

[11]Jiang T., Yang Y., Song Y., "Exponential companding transform for PAPR reduction in OFDM systems", IEEE Transaction on Broadcasting, vol. 51, No. 2, pp. 244-248, June 2005. 